\title{
The Role of Radiotherapy in Endometrial Cancer: Current Evidence and Trends
}

\author{
Carien L. Creutzberg $\cdot$ Remi A. Nout
}

Published online: 16 August 2011

(C) The Author(s) 2011. This article is published with open access at Springerlink.com

\begin{abstract}
Adjuvant treatment of patients with endometrial cancer is tailored to clinical-pathological prognostic factors. Pelvic radiation therapy for stage I endometrial cancer (EC) provides a highly significant improvement of local control, but without survival advantage. Low-risk EC patients have a very favorable prognosis, and should be observed after surgery. Use of adjuvant radiotherapy (RT) is limited to patients with high-intermediate or high-risk factors. For those with high-intermediate risk features, vaginal brachytherapy alone provides excellent vaginal control with less morbidity and better quality of life than pelvic external beam RT (EBRT). For patients with stage I-III EC with high-risk features, the use of adjuvant chemotherapy alone has not shown survival benefit as compared to pelvic EBRT. A first trial comparing pelvic EBRT with or without adjuvant chemotherapy has shown better progression-free survival with combined therapy. Current ongoing trials are exploring the role of combined RT and chemotherapy, compared to chemotherapy or RT alone.
\end{abstract}

Keywords Endometrial cancer-Adjuvant therapy.

Radiation therapy Prognostic factors · Side effects .

Quality of life

\section{Introduction}

Endometrial cancer (EC) is the most common gynecological cancer and primarily affects postmenopausal

C. L. Creutzberg $(\square) \cdot$ R. A. Nout

Department of Clinical Oncology, postzone K1-P,

Leiden University Medical Center,

Albinusdreef 2, P.O. Box 9600, 2300 RC Leiden,

The Netherlands

e-mail: c.l.creutzberg@lumc.nl women between 60 and 85 years of age. Many patients have concurrent comorbidities, such as obesity, diabetes, and cardiovascular diseases. Annual incidence rates in European countries range between 15 and 20 per 100,000 women; incidence in the United States is 23 per $10^{5}$ women per year $[1,2]$.

The large majority of patients are diagnosed at early stage (International Federation of Gynecology and Obstetrics [FIGO] stage I), due to early symptoms. Surgery, consisting of total abdominal hysterectomy and bilateral salpingo-oophorectomy (TAH-BSO), is the primary treatment. More recently, laparoscopic surgery (laparoscopicassisted vaginal hysterectomy or total laparoscopic hysterectomy) has been introduced for early-stage disease. The advantages of laparoscopy are the shorter hospitalization and recovery time and decrease of surgical morbidity. Disadvantages are the increased length of the operation and the learning curve involved with laparoscopic techniques. First results from randomized trials comparing laparoscopic surgery to abdominal surgery have shown the overall (OS) and relapse-free survival (RFS) rates to be similar to laparotomy [3], with shorter hospital stay, more rapid recovery, and less pain, while complication rates were similar [4]. Short-term quality of life (up to 4 weeks postsurgery), especially physical functional well being, was better after laparoscopy [5].

The role of pelvic and para-aortic lymphadenectomy or lymph node sampling has been widely debated. Two large randomized trials have been published, both showing no differences in overall or progression-free survival rates between the arms with and without lymphadenectomy (LA) for patients with stage I EC. Although LA did identify $13 \%$ microscopic nodal metastases in the LA group, as compared to $3 \%$ in the no-LA group, survival and patterns of relapse were identical in the two groups $[6,7]$. 
Definitive staging according to the FIGO system is based on surgical and pathology findings. In 2009, the revised FIGO staging system was published, which replaced the 1988 FIGO staging system [8]. Especially the changes in stage IA, IB, and IC should be kept in mind when evaluating literature data, as FIGO 1988 stage IA and IB have been grouped together in FIGO 2009 as stage IA, and FIGO 1988 stage IC is IB in FIGO 2009 [8].

\section{Risk Groups and Adjuvant Radiation Therapy}

Major prognostic factors for endometrial carcinoma are stage, age, histological type, grade, depth of myometrial invasion, and presence of lymph-vascular space invasion (LVSI). Adjuvant radiotherapy (RT) for endometrial carcinoma has increasingly been tailored to these risk factors. Based on staging studies and prospective and retrospective data, endometrial carcinoma has been classified as low-risk, intermediate-risk, and high-risk for lymph node metastases and/or early disease spread to the abdominal cavity and to distant sites. The majority of patients with EC have low to intermediate $(55 \%)$ or high-intermediate (30\%) risk features; only $15 \%$ have a high-risk profile. FIGO staging was updated in 2009; risk profiles have been based on the 1988 FIGO stage but have been updated to FIGO 2009 criteria. Low-risk are FIGO 2009 [8] stage IA (with no or superficial $[<50 \%]$ myometrial invasion) EC, grade 1 or 2, of endometrioid type histology. High-risk are FIGO stage IB (ie, outer [ $>50 \%]$ myometrial invasion) of grade 3 or of non-endometrioid histology; or stage II or III EC. All other stage I EC are intermediate risk; this group has further been refined with other factors to define a high-intermediate risk (HIR) group [9, 10]. Five-year survival rates for patients with intermediate-risk EC are 80
$85 \%$, with most of these patients dying of intercurrent diseases; rates of endometrial cancer death are $8-10 \%$.

For low-risk EC standard treatment is surgery alone, with $95 \%$ probability of 5 -year RFS. For intermediaterisk EC, four large randomized trials have established the role of adjuvant external beam pelvic radiotherapy (EBRT; Table 1) [9-11, 12••]. The Norwegian trial, published in 1980, included 540 women with clinical stage 1 endometrial carcinoma [11]. After hysterectomy and postoperative vaginal brachytherapy (60 Gy to the mucosal surface), patients were randomly assigned to additional EBRT (40 Gy in 2 Gy fractions) or observation. Although additional EBRT reduced vaginal and pelvic relapse rates ( $2 \%$ at 5 years vs $7 \%$ in the control group), more distant metastases were found in the RT group $(10 \%$ vs $5 \%$ ), and survival was not improved ( $89 \%$ vs $91 \%$ at 5 years). The subgroup with grade 3 tumors with deep $(>50 \%)$ myometrial invasion showed improved local control and survival after EBRT (18\% vs $27 \%$ cancerrelated deaths); however, there were too few patients in this category to reach significance.

In the first Post-Operative Radiation Therapy in Endometrial Carcinoma (PORTEC) trial, 715 patients with stage I endometrial carcinoma, grade 1 or 2 with deep $(\geq 50 \%)$ myometrial invasion or grade 2 or 3 with superficial $(<50 \%)$ invasion, were randomized after TAH-BSO to receive EBRT (46 Gy in 2 Gy fractions) or no additional treatment (NAT) [9]. The 10-year locoregional relapse rates were $5 \%$ in the EBRT group and $14 \%$ in the control group $(P<0.0001)$. There was no significant survival difference between the treatment arms, with 10-year overall survival of $68 \%$ (EBRT) vs $73 \%$ (NAT, $P=0.14$ ) and endometrial cancer-related death rates of $10 \%$ and $8 \%(P=0.47)$ [13]. Risk criteria for locoregional relapse were grade 3 , age older than 60 years, and outer $50 \%$ invasion.

Table 1 Randomized trials of adjuvant radiation therapy in stage I endometrial carcinoma

\begin{tabular}{|c|c|c|c|c|c|}
\hline Trial (ref) accrual period & No. patients eligibility & Surgery & Randomization & $\begin{array}{l}\text { Locoregional } \\
\text { recurrence }\end{array}$ & Survival \\
\hline $\begin{array}{c}\text { Norwegian [11] } \\
1968-1974\end{array}$ & 540 Stage I & TAH-BSO & $\begin{array}{l}\text { VBT vs VBT + } \\
\text { pelvic RT }\end{array}$ & $\begin{array}{l}7 \% \text { vs } 2 \% \text { at } 5 \text { years } \\
P<0.01\end{array}$ & $\begin{array}{c}89 \% \text { vs } 91 \% \text { at } \\
5 \text { years } P=\mathrm{NS}\end{array}$ \\
\hline $\begin{array}{c}\text { PORTEC-1 [9] } \\
\text { 1990-1997 }\end{array}$ & $\begin{array}{l}714 \text { IB grade } 2-3 \text { IC } \\
\text { grade } 1-2\end{array}$ & TAH-BSO & NAT vs pelvic RT & $\begin{array}{l}14 \% \text { vs } 4 \% \text { at } 5 \text { years } \\
P<0.001\end{array}$ & $\begin{array}{l}85 \% \text { vs } 81 \% \text { at } \\
5 \text { years } P=0.31\end{array}$ \\
\hline $\begin{array}{c}\text { GOG-99 [10] } \\
1987-1995\end{array}$ & $\begin{array}{l}392 \text { Stage IB, IC } \\
\text { Stage II (occult) }\end{array}$ & $\begin{array}{l}\text { TAH-BSO and } \\
\text { lymphadenectomy }\end{array}$ & NAT vs pelvic RT & $\begin{array}{l}12 \% \text { vs } 3 \% \text { at } 2 \text { years } \\
P<0.01\end{array}$ & $\begin{array}{l}86 \% \text { vs } 92 \% \text { at } \\
4 \text { years } P=0.56\end{array}$ \\
\hline $\begin{array}{l}\text { ASTEC/EN5 [12••] } \\
\text { 1996-2005 }\end{array}$ & $\begin{array}{l}905 \text { Stage IAB g3, IC, } \\
\text { Stage II, serous/cc }\end{array}$ & $\begin{array}{l}\text { TAH-BSO } \pm \\
\text { lymphadenectomy }\end{array}$ & NAT vs pelvic RT & $\begin{array}{c}7 \%{ }^{\mathrm{a}} \text { vs } 4 \% \text { at } 5 \text { years } \\
P=0.038\end{array}$ & $\begin{array}{l}84 \% \text { vs } 84 \% \text { at } \\
5 \text { years } P=0.98\end{array}$ \\
\hline $\begin{array}{l}\text { PORTEC-2 }[21 \bullet \bullet] \\
\text { 2002-2006 }\end{array}$ & $\begin{array}{l}427, \text { age }>60 \text { IB grade } 3 \\
\text { IC grade } 1-2\end{array}$ & TAH-BSO & VBT vs pelvic RT & $\begin{array}{l}2 \% \text { vs } 2 \% \text { at } 5 \text { years } \\
\quad P=0.74\end{array}$ & $\begin{array}{l}85 \% \text { vs } 80 \% \text { at } \\
5 \text { years } P=0.57\end{array}$ \\
\hline
\end{tabular}

${ }^{\text {a }} 53 \%$ in NAT arm received VBT; isolated locoregional recurrence reported

$N A T$ no additional treatment; $R T$ radiation therapy; $V B T$ vaginal brachytherapy 
Five-year survival after any relapse was $12 \%$ in the RT group and $48 \%$ in the control group $(P<0.001)$ [14]. This was due to the fact that $75 \%$ of locoregional relapses in the NAT group were located in the vagina. After vaginal relapse, 5-year actuarial survival was $64 \% ; 38 \%$ in the EBRT group versus $70 \%$ in the NAT group, which shows the high salvage rates of vaginal relapse in patients not previously irradiated. In contrast, outcome after pelvic and distant relapse was poor, with only $11 \% 5$-year survival.

The GOG\#99 trial included 392 evaluable patients with FIGO 1988 stage IB, IC, or IIA endometrial carcinoma of any histological grade, who were randomized after TAHBSO and lymphadenectomy to receive pelvic EBRT (50.4 Gy in 1.8 Gy fractions) or NAT [10]. A highintermediate risk group (HIR, 33\% of the study patients) was defined based on the prognostic factors age, histological grade, myometrial invasion, and the presence of lymphovascular space invasion. RT resulted in similar hazard reductions for the HIR and LIR subgroups (58\% and $54 \%$ ), but in absolute terms, the differences were greater for HIR patients, with a reduction of 4-year cumulative relapse from $27 \%$ (NAT) to $13 \%$ (RT). The 2year estimated vaginal and pelvic failure rate was $12 \%$ in the NAT group and 3\% in the EBRT group, for a $58 \%$ hazard reduction by RT. These results are strikingly similar to those obtained in the PORTEC study without lymphadenectomy. However, the 4-year crude rate of severe complications in GOG-99 was $13 \%$, which underlines the increased risk of toxicity when combining extended surgery with pelvic radiotherapy. In addition, GOG\#99 has shown that the HIR factors are associated with increased risk of relapse, regardless of lymphadenectomy, and that LVSI is strongly associated with risk of lymph node involvement, as well as risk of relapse at distant sites and inferior outcome in absence of lymph node metastases [10, 15, 16].

In the pooled ASTEC and EN5 trials, 905 patients with stage I endometrial carcinoma with risk features (deep invasion or high grade) were randomly allocated to EBRT or NAT $[12 \bullet \cdot]$. There was no difference in overall survival $(84 \%$ at 5 years in both groups), confirming the results of the PORTEC and GOG\#99 trials. In the ASTEC/EN5 trial, brachytherapy was used at discretion of the centers and was used in both arms. As a consequence, $51 \%$ of the patients in the NAT arm received vaginal brachytherapy. This can explain the fact that the 5-year rate of isolated (not total) vaginal or pelvic recurrence was $6.1 \%$ in the NAT arm, compared to $3.2 \%$ in the EBRT arm (hazard ratio $0.46, P=-0.02$ ).

Conclusions from these randomized trials are that EBRT provides a highly significant improvement of local control, but without survival advantage. Furthermore, mild adverse effects were recorded in $26 \%$ of EBRT patients in the PORTEC-1 trial, predominantly gastrointestinal (GI) toxicity [17]. A large proportion of endometrial cancer patients has a very favorable prognosis, and should be observed after TAH-BSO. Radiation therapy is a very effective salvage treatment for vaginal relapse in patients not previously irradiated. The use of postoperative RT should therefore be limited to the group of patients at sufficiently high risk of locoregional relapse to warrant the risk of treatment-associated morbidity. The 10-year locoregional relapse rates in the PORTEC-HIR group were $4.6 \%$ in the RT group and $23.1 \%$ in the control group [13]. In the GOG99 trial, EBRT provided a reduction of isolated 4-year local relapse in the HIR group from $13 \%$ to $5 \%$.

\section{Adjuvant Vaginal Brachytherapy Alone}

The results of the randomized trials for intermediate-risk endometrial carcinoma suggested that, in view of the absence of survival benefit with EBRT and of the fact that most $(75 \%)$ locoregional recurrences were located in the vagina, vaginal brachytherapy (VBT) might also be effective for patients with high-intermediate risk features to obtain local control with fewer side effects than EBRT and better quality of life. Data from retrospective studies that used VBT alone for stage I, mainly low-risk EC showed 5-year rates of vaginal relapse of $0-5 \%$ [18-20]. This was the rationale for the randomized PORTEC-2 trial, which compared EBRT and VBT with regard to efficacy, side effects, and health-related quality of life (HRQL). In the PORTEC-2 trial, 427 patients with FIGO 1988 stage IIIA endometrial carcinoma with high-intermediate risk features (age of at least 60 years, grade 1 or 2 tumors with outer $50 \%$ invasion or grade 3 with inner $50 \%$ invasion) were randomly assigned after surgery (TAH-BSO) to EBRT $(n=214)$ or VBT $(n=213)$. Final results of the PORTEC-2 trial showed vaginal brachytherapy to be highly effective in preventing vaginal relapse. At median follow-up of 45 months, estimated 5-year rates of vaginal recurrence were $1.8 \%$ for VBT and $1.6 \%$ for EBRT $(P=0.74)$. Fiveyear rates of locoregional relapse (VR and/or PR) were $5.1 \%$ and $2.1 \%(P=0.17)$. Only $1.5 \%$ versus $0.5 \%(P=0.30)$ presented with isolated pelvic recurrence $(\mathrm{PR})$; other PR were part of widespread disease relapse. Rates of DM were similar $(8.3 \%$ vs $5.7 \%, P=0.46)$. There were no differences in OS $(84.8 \%$ vs $79.6 \%, P=0.57)$ and DFS $(82.7 \%$ vs $78.1 \%, P=0.74)$ between the groups. Rates of grade $1-2$ gastrointestinal toxicity were significantly lower in the VBT group $[21 \bullet \bullet]$. Quality of life was significantly better in the VBT arm. Patients who had brachytherapy reported better social functioning $(P<0.002)$ and lower symptom scores for diarrhea, fecal leakage, the need to stay close to the toilet, and limitation in daily activities due to bowel symptoms $(P<0.001)$. Sexual functioning and symptoms did not differ between the treatment arms [22•]. In view of 
the efficacy of VBT with fewer side effects and better quality of life, VBT alone has in many countries become standard of care for patients with EC with high-intermediate risk features.

\section{Adjuvant Chemotherapy for High-Risk and Advanced-Stage EC}

Patients with high-risk endometrial cancer (FIGO 2009 stage IB grade 3, stages II-III, and those with serous or clear cell cancers with myometrial invasion) have an increased risk of distant metastases and endometrial cancer death, and the role of adjuvant chemotherapy has been investigated in several trials (Table 2). Three randomized trials have investigated use of adjuvant chemotherapy as compared to radiation therapy [2325]. Both the Japanese and Italian trials used cyclophosphamide, doxorubicin, and cisplatin (CAP) chemotherapy (3 or 5 cycles, respectively), randomly compared to pelvic EBRT alone. In the Japanese trial (385 patients) [23], no differences in overall survival, relapse rate, or progression-free survival were found. Grade 3-4 toxicity was reported in $4.7 \%$ (CAP) versus $1.6 \%$ (EBRT) of the patients. The Italian trial (345 patients) included $65 \%$ patients with stage III EC, reflected in 5 -year overall survival of $69 \%$ vs $66 \%(P=0.77)$ [24]. There were no differences in 5-year OS, PFS, or RR. More grade 3 toxicity was reported in the CAP arm (39\% hematologic, $10 \%$ nausea/vomiting) than in the EBRT arm (16\%, mainly GI toxicity). In the GOG\#122 trial, 396 patients with advanced disease (stage III and IV EC) were included [25]. This trial allowed residual macroscopic disease up to $2 \mathrm{~cm}$, and used prolonged chemotherapy, 8 cycles of doxorubicin and cisplatin (AP) in one arm; and whole-abdominal radiation therapy (WART) in the second arm. Predicted 5-year survival, adjusted for stage, was significantly better for AP $(55 \%$ vs $42 \%$ ), both for the whole study population and for the $73 \%$ stage III patients, but toxicity was substantial: grade 3-4 GI toxicity $20 \%$ versus $13 \%$, and neurologic toxicity $7 \%$ versus $1 \% ; 17 \%$ of patients did not complete AP in view of toxicity. Event rates were similar ( $54 \%$ vs $50 \%$ ), and unadjusted predicted 5-year PFS rates were $42 \%$ versus $38 \%$. WART cannot be recommended for treatment of abdominal disease spread.

\section{Role of Combined Radiation Therapy and Chemotherapy}

The trials comparing adjuvant chemotherapy (CT) alone and adjuvant EBRT alone showed that while chemotherapy delayed distant relapse, pelvic EBRT delayed pelvic relapse and the overall and relapse-free survival rates were similar. Pelvic EBRT is still regarded an essential treatment component

Table 2 Randomized trials investigating adjuvant chemotherapy and/or radiotherapy in endometrial cancer

\begin{tabular}{|c|c|c|c|c|}
\hline Trial (ref) & No. patients eligibility/stage & Randomization & Pelvic recurrence & Overall survival \\
\hline Susumu et al. [23] & $\begin{array}{l}385 \text { Stage I-III with }>50 \% \\
\text { MI }(60 \% \text { stage IB })\end{array}$ & $\begin{array}{l}\text { Pelvic RT vs chemotherapy } \\
(3 \times \text { CAP })\end{array}$ & $7 \%$ vs $7 \% P=\mathrm{ns}$ & $85 \%$ vs $87 \% P=\mathrm{ns}$ \\
\hline Maggi et al. [24] & 345 IB-II grade $3(35 \%)$ III (65\%) & $\begin{array}{l}\text { Pelvic RT vs chemotherapy } \\
(5 \times \text { CAP })\end{array}$ & $12 \%$ vs $16 \% P=\mathrm{ns}$ & $69 \%$ vs $66 \% P=\mathrm{ns}$ \\
\hline Randall et al. [25] & 396 III; IV (28\%) (residual <2 cm) & $\begin{array}{l}\text { WART vs chemotherapy } \\
(8 \times \mathrm{AP})\end{array}$ & $13 \%$ vs $18 \%$ & $42 \%$ vs $53 \% P<0.01$ \\
\hline Morrow et al. [28] & $\begin{array}{l}181 \text { Clinical stage I II (occult) } \\
31 \% \text { node }+\end{array}$ & $\begin{array}{l}\text { Pelvic RT vs pelvic RT and } \\
\text { chemotherapy }(6-8 \times \mathrm{A})\end{array}$ & N/A & No difference $P=\mathrm{ns}$ \\
\hline Kuoppala et al. [29] & $\begin{array}{l}156 \text { Stage IA g3, IB, } \\
\text { Stage II-IIIA ( } 46 \% \text { stage IB) }\end{array}$ & $\begin{array}{l}\text { Pelvic RT vs pelvic RT and } \\
\text { chemotherapy }(3 \times \text { CEP })\end{array}$ & $3 \%$ vs $2 \% P=\mathrm{ns}$ & $\begin{array}{c}85 \% \text { vs } 82 \% \text { DSS } \\
5 \text { years } P=\mathrm{ns}\end{array}$ \\
\hline Hogberg et al. $[30 \bullet \bullet]$ & $\begin{array}{l}382 \text { Stage I-III Stage I } \\
\text { serous ( } 49 \% \text { stage IB) }\end{array}$ & $\begin{array}{l}\text { Pelvic RT vs pelvic RT and } \\
\text { chemotherapy } \\
(4 \times \text { AP/TAP/TC/TEP })\end{array}$ & N/A & $76 \%$ vs $83 \% P=\mathrm{ns}$ \\
\hline Ongoing trials & Planned no. & Randomization & \multicolumn{2}{|c|}{ Accrual January 2011} \\
\hline PORTEC-3 & $\begin{array}{l}670 \text { Stages I-III with } \\
\text { high-risk factors; serous/cc }\end{array}$ & $\begin{array}{l}\text { Pelvic RT vs RT-CT } \\
(2 \times \mathrm{C} \text { during } \mathrm{RT} \text { and } 4 \times \mathrm{TC})\end{array}$ & \multicolumn{2}{|l|}{300} \\
\hline GOG\#249 & $\begin{array}{l}562 \text { Stages I-II with } \\
\text { high-risk factors or serous/cc }\end{array}$ & $\begin{array}{l}\text { Pelvic RT vs VBT and } \\
\text { CT }(3 \times \text { TC })\end{array}$ & \multicolumn{2}{|l|}{175} \\
\hline GOG\#258 & 804 Stages III/IV & $\begin{array}{l}\text { RT-CT }(2 \times \mathrm{C} \text { during } \mathrm{RT} \text { and } 4 \times \mathrm{TC}) \\
\text { vs CT }(6 \times \mathrm{TC})\end{array}$ & \multicolumn{2}{|l|}{132} \\
\hline
\end{tabular}

$A$ doxorubicin; $A P$ doxorubicin/cisplatin; $C A P$ cyclophosphamide/doxorubicin/cisplatin; $C E P$ cyclophosphamide/epirubicin/cisplatin; $C T$ chemotherapy; $n s$ not statistically significant; $R T$ radiation therapy; TAP paclitaxel/doxorubicin/cisplatin; $T C$ paclitaxel/carboplatin; $T E P$ paclitaxel/epirubicin/cisplatin; WART whole abdominal radiotherapy 
for patients with high-risk or advanced-stage EC. Retrospective studies reported significantly higher rates of pelvic recurrence if high-risk patients were treated without RT [26, 27]. In a retrospective analysis of 43 patients with high-risk or advanced-stage endometrial carcinoma who received chemotherapy alone, $67 \%$ relapsed, of whom $40 \%$ had pelvic recurrence and $56 \%$ distant relapse. The 3 -year pelvic failure rate was $47 \%$, and in $31 \%$ the pelvis was the first or only site of recurrence [26]. In an analysis of 71 stage IIIC EC patients, those treated with regional RT had a significantly better 5 -year pelvic-relapse-free survival than patients who received CT alone ( $98 \%$ vs $61 \%, P=0.001)$, as well as better disease-specific survival ( $78 \%$ vs $39 \%, P=0.01$ ), and overall survival ( $73 \%$ vs $40 \%, P=0.03$ ). In patients treated without regional RT, the most common site of relapse was the pelvis [27].

The way forward might be to combine CT and RT. This has first been investigated in a small GOG trial (181 patients, closed early and flawed by many patients not receiving chemotherapy) in which patients were assigned to RT with or without single-agent doxorubicin. This trial did not show any difference in survival or recurrence patterns [28]. A Finnish trial was also underpowered, with 156 patients randomized to EBRT with or without 3 cycles of cisplatin, epirubicin, and cyclophosphamide (CEP); mainly intermediate-risk patients. This trial showed no differences in the rates of survival $(P=0.77)$ or recurrence (RT vs $\mathrm{RT}+$ CT: $18 \%$ vs $22 \%$ ), but more toxicity in the $\mathrm{RT}+\mathrm{CT}$ arm (severe bowel toxicity $9.5 \%$ vs $2.8 \%$ ) [29].

The most recent and largest trial, NSGO 9501/EORTC 55991, randomly compared EBRT with or without four cycles of CT in 382 patients. Various chemotherapy combinations were used, mainly doxorubicin and cisplatin (AP), but also AP with paclitaxel (TAP), cisplatin or carboplatin with paclitaxel (TP or TC), and TP with epirubicin (TEP), given before or after RT. This trial was the first to show a significant $7 \%$ increase in progressionfree survival with the addition of chemotherapy ( $79 \%$ vs $72 \%, P=0.03$ ), but no significant difference in overall survival. In the published pooled data analysis with the Italian MaNGO ILIADE-III trial (157 patients, for a combined total of 534 evaluable patients), results were similar, with a statistically significant difference in 5-year PFS favoring the combined arm $(78 \%$ vs $69 \%, P=0.009)$, but only a trend for improved 5 -year OS $(82 \%$ vs $75 \%, P=$ 0.07 ) [30]. It should be noted that although NSGO/EORTC and GOG122 trials both showed PFS benefit with chemotherapy for the overall trial populations, neither showed a trend for PFS or OS benefit from adjuvant chemotherapy for the subpopulations of patients with serous and clear cell tumors, although these were few in number $(20 \%, n=140$ in NSGO/EORTC and $n=100$ in GOG\#122). The hazard ratios for PFS and OS with chemotherapy were 0.91 and
1.02 for patients with serous cancer in GOG\#122, and 0.83 and 0.94 , respectively, for patients with serous cancer in the NSGO/EORTC trial $[25,30 \bullet]$. This underlines the need to establish the efficacy of adjuvant chemotherapy for serous cancers in randomized trials.

\section{Current Randomized Trials}

A number of trials are currently ongoing which further explore the role of concurrent chemoradiation and/or adjuvant chemotherapy. These trials are aimed at answering a number of unresolved issues in the treatment of patients with high-risk EC, such as the role of EBRT or VBT in combination with chemotherapy; the question if overall survival benefit can be obtained in these elderly patients without impacting too strongly on their quality of life; if chemotherapy benefits patients with serous or clear cell cancers; and if combined treatment should be given to all patients with high risk factors or only to those with advanced disease.

The international randomized PORTEC-3 trial for patients with high-risk and advanced-stage disease compares pelvic EBRT alone with combined RT-CT (concurrent chemoradiation with two cycles of cisplatin during RT, followed by four adjuvant cycles of carboplatin and paclitaxel), to establish if RT-CT improves overall and failure-free survival rates, and the cost/benefit ratio in terms of toxicity and quality of life. This trial uses a uniform treatment schedule starting both treatment modalities early, includes upfront pathology review to ensure that only true high-risk patients are included, and quality of life assessments to establish short-term and long-term toxicities and their impact on the patients' daily life. With international intergroup collaboration patient accrual is well underway.

The randomized GOG\#249 trial for patients with stage I-II EC with high-intermediate or high-risk factors compares pelvic EBRT alone, with vaginal brachytherapy followed by adjuvant chemotherapy (three cycles of carboplatin and paclitaxel). The randomized GOG\#258 trial includes patients with stage III-IVA EC and compares the same RT-CT schedule as used in the PORTEC-3 trial with chemotherapy alone (6 cycles of carboplatin and paclitaxel). These GOG trials are also accruing well (Table 2).

All of these trials are better powered than their predecessors, and should help resolve many of the uncertainties regarding which patients may benefit from chemotherapy and/or radiotherapy.

\section{Conclusions}

Over the past decades, randomized trials have established the role of adjuvant RT for endometrial cancer. The use of 
external beam RT has become limited to EC patients with high-risk factors, sparing many the toxicities and impact on long-term quality of life of EBRT. Adjuvant brachytherapy alone has become the standard of care for patients with high-intermediate risk factors. Randomized trials comparing EBRT and chemotherapy for high-risk EC have not shown overall survival improvement with chemotherapy alone; the first trial comparing combined RT and CT has shown improved progression-free survival compared to RT alone. Current trials are exploring the roles of concurrent chemoradiation and/or adjuvant chemotherapy compared to CT or RT alone. These trials will resolve many of the uncertainties regarding which patients may benefit from chemotherapy and/or radiotherapy.

Disclosure No potential conflicts of interest relevant to this article were reported.

Open Access This article is distributed under the terms of the Creative Commons Attribution Noncommercial License which permits any noncommercial use, distribution, and reproduction in any medium, provided the original author(s) and source are credited.

\section{References}

Papers of particular interest, published recently, have been highlighted as:

- Of importance

-• Of major importance

1. Dutch Cancer Registry. Dutch Cancer Registry, http://www.ikcnet. $\mathrm{nl} /$ cijfers.

2. Altekruse SF, Kosary CL, Krapcho M, Neyman N, Aminou R, Waldron W, Ruhl J, Howlader N, Tatalovich Z, Cho H, Mariotto A, Eisner MP, Lewis DR, Cronin K, Chen HS, Feuer EJ, Stinchcomb DG, Edwards BK (eds). SEER Cancer statistics review, 1975-2007. 2010. National Cancer Institute. Bethesda, MD. http://seer.cancer.gov/csr/1975 2007/, based on November 2009 SEER data submission, posted to the SEER web site, 2010.

3. Malzoni M, Tinelli R, Cosentino F, et al. Total laparoscopic hysterectomy versus abdominal hysterectomy with lymphadenectomy for early-stage endometrial cancer: a prospective randomized study. Gynecol Oncol. 2009;112:126-33.

4. Mourits MJ, Bijen CB, Arts HJ, et al. Safety of laparoscopy versus laparotomy in early-stage endometrial cancer: a randomised trial. Lancet Oncol. 2010;11:763-71.

5. Janda M, Gebski V, Brand A, et al. Quality of life after total laparoscopic hysterectomy versus total abdominal hysterectomy for stage I endometrial cancer (LACE): a randomised trial. Lancet Oncol. 2010;11:772-80.

6. Kitchener H, Swart AM, Qian Q, et al. Efficacy of systematic pelvic lymphadenectomy in endometrial cancer (MRC ASTEC trial): a randomised study. Lancet. 2009;373:125-36.

7. Benedetti PP, Basile S, Maneschi F, et al. Systematic pelvic lymphadenectomy vs. no lymphadenectomy in early-stage endo- metrial carcinoma: randomized clinical trial. J Natl Cancer Inst. 2008;100:1707-16.

8. Pecorelli S. Revised FIGO staging for carcinoma of the vulva, cervix, and endometrium. Int J Gynaecol Obstet. 2009;105:103-4.

9. Creutzberg CL, van Putten WL, Koper PC, et al. Surgery and postoperative radiotherapy versus surgery alone for patients with stage-1 endometrial carcinoma: multicentre randomised trial. PORTEC Study Group. Post operative radiation therapy in endometrial carcinoma. Lancet. 2000;355:1404-11.

10. Keys HM, Roberts JA, Brunetto VL, et al. A phase III trial of surgery with or without adjunctive external pelvic radiation therapy in intermediate risk endometrial adenocarcinoma: a Gynecologic Oncology Group study. Gynecol Oncol. 2004;92:744-51.

11. Aalders J, Abeler V, Kolstad P, et al. Postoperative external irradiation and prognostic parameters in stage I endometrial carcinoma: clinical and histopathologic study of 540 patients. Obstet Gynecol. 1980;56:419-27.

12. • Blake P, Swart AM, Orton J, et al. Adjuvant external beam radiotherapy in the treatment of endometrial cancer (MRC ASTEC and NCIC CTG EN.5 randomised trials): pooled trial results, systematic review, and meta-analysis. Lancet. 2009;373:137-46. Largest randomized trial comparing pelvic EBRT to no adjuvant treatment after surgery for stage I EC.

13. Scholten AN, van Putten WL, Beerman H, et al. Postoperative radiotherapy for Stage 1 endometrial carcinoma: long-term outcome of the randomized PORTEC trial with central pathology review. Int J Radiat Oncol Biol Phys. 2005;63:834-8.

14. Creutzberg CL, van Putten WLJ, Koper PC, et al. Survival after relapse in patients with endometrial cancer: results from a randomized trial. Gynecol Oncol. 2003;89:201-9.

15. Cohn DE, Horowitz NS, Mutch DG, et al. Should the presence of lymphvascular space involvement be used to assign patients to adjuvant therapy following hysterectomy for unstaged endometrial cancer? Gynecol Oncol. 2002;87:243-6.

16. Briet JM, Hollema H, Reesink N, et al. Lymphvascular space involvement: an independent prognostic factor in endometrial cancer. Gynecol Oncol. 2005;96:799-804.

17. Creutzberg CL, van Putten WL, Koper PC, et al. The morbidity of treatment for patients with Stage I endometrial cancer: results from a randomized trial. Int J Radiat Oncol Biol Phys. 2001;51:1246-55.

18. Rittenberg PV, Lotocki RJ, Heywood MS, et al. High-risk surgical stage 1 endometrial cancer: outcomes with vault brachytherapy alone. Gynecol Oncol. 2003;89:288-94.

19. Weiss E, Hirnle P, Arnold-Bofinger H, et al. Adjuvant vaginal high-dose-rate afterloading alone in endometrial carcinoma: patterns of relapse and side effects following low-dose therapy. Gynecol Oncol. 1998;71:72-6.

20. Pearcey RG, Petereit DG. Post-operative high dose rate brachytherapy in patients with low to intermediate risk endometrial cancer. Radiother Oncol. 2000;56:17-22.

21. • Nout RA, Smit VTHB, Putter H, et al. Vaginal brachytherapy versus pelvic external beam radiotherapy for patients with endometrial carcinoma of high-intermediate risk (PORTEC-2): an open-label, non-inferiority, randomised trial. Lancet.2010;375:816-23. First randomized trial comparing adjuvant pelvic $R T$ and vaginal brachytherapy for EC with HIR features.

22. - Nout RA, Putter H, Jurgenliemk-Schulz IM, et al. Quality of life after pelvic radiotherapy or vaginal brachytherapy for endometrial cancer: first results of the randomized PORTEC-2 trial. J Clin Oncol. 2009;27:3547-56. Extensive analysis of quality of life in the PORTEC trial, showing difference between pelvic RT and vaginal brachytherapy.

23. Susumu N, Sagae S, Udagawa Y, et al. Randomized phase III trial of pelvic radiotherapy versus cisplatin-based combined chemotherapy in patients with intermediate- and high-risk endometrial 
cancer: a Japanese Gynecologic Oncology Group study. Gynecol Oncol. 2008;108:226-33.

24. Maggi R, Lissoni A, Spina F, et al. Adjuvant chemotherapy vs radiotherapy in high-risk endometrial carcinoma: results of a randomised trial. Br J Cancer. 2006;95:266-71.

25. Randall ME, Filiaci VL, Muss H, et al. Randomized phase III trial of whole-abdominal irradiation versus doxorubicin and cisplatin chemotherapy in advanced endometrial carcinoma: a Gynecologic Oncology Group Study. J Clin Oncol. 2006;24:36-44.

26. Mundt AJ, McBride R, Rotmensch J, et al. Significant pelvic recurrence in high-risk pathologic stage I-IV endometrial carcinoma patients after adjuvant chemotherapy alone: implications for adjuvant radiation therapy. Int $\mathrm{J}$ Radiat Oncol Biol Phys. 2001;50:1145-53.

27. Klopp AH, Jhingran A, Ramondetta L, et al. Node-positive adenocarcinoma of the endometrium: outcome and patterns of recurrence with and without external beam irradiation. Gynecol Oncol. 2009;115:6-11.

28. Morrow CP, Bundy BN, Homesley HD, et al. Doxorubicin as an adjuvant following surgery and radiation therapy in patients with high-risk endometrial carcinoma, stage I and occult stage II: a Gynecologic Oncology Group Study. Gynecol Oncol. 1990;36:166-71.

29. Kuoppala T, Maenpaa J, Tomas E, et al. Surgically staged high-risk endometrial cancer: randomized study of adjuvant radiotherapy alone vs. sequential chemo-radiotherapy. Gynecol Oncol. 2008;110:190-5.

30. • Hogberg T, Signorelli M, De Oliveira CF, et al. Sequential adjuvant chemotherapy and radiotherapy in endometrial cancerresults from two randomised studies. Eur J Cancer.2010;46:242231. This is the first randomized trial comparing RT alone with combination of RT and CT, showing significant PFS but not OS improvement. 